\title{
A supplementary report on the toxic manifestations of CHOP regimen in subjects with intermediate grade NHL
}

\author{
*Ambili Remesh \\ Department of Pharmacology, Dr. Somervell Memorial CSI Medical College, Karakonam, Trivandrum, Kerala, India
}

\begin{abstract}
Cancer chemotherapy engenders a gamut of unpredictable and perilous toxicities, which can cause substantial mortality, morbidity, and results in escalated healthcare costs due to increased requirement for ameliorative treatments and toxicity-related hospitalization. The main objective of this article is to report a range of adverse effects of $\mathrm{CHOP}$ regimen in patients with intermediate grade Non-Hodgkin's Lymphoma (NHL). This randomized prospective study was conducted on de novo intermediate grade NHL patients, aged $>20 \leq 60$ years, receiving CHOP chemotherapy regimen. Out of 30 patients who satisfied the inclusion criteria, only 25 patients completed 6 cycles of chemotherapy and were evaluated for various toxicities. Evaluation of all the 25 subjects showed that, in comparison with the baseline, most patients exhibited toxic damages. The hepatopancreatic, dermatological, vascular and nephrological damages, eventually resulted in weight loss, hepatomegaly, alopecia, hypotension, as well as abnormal levels of serum liver markers, serum creatinine and blood glucose. But, the necessity to interrupt the CHOP treatment did not arise in any of the patients. Thus, in this study combination chemotherapy with CHOP protocol was well tolerated by majority of the subjects.
\end{abstract}

Key Words: Intermediate grade NHL, CHOP chemotherapy, toxicity profile.

\section{INTRODUCTION}

The widespread occurrence of Non-Hodgkin's lymphoma (NHL) makes it the most common hematologic cancer among adults in the world. NHL constitutes a heterogeneous group of malignant neoplasms of the lymph nodes and lymphoid tissues such as spleen, bone marrow, and other organs of the immune system (van de Schans et al., 2011). NHL is characterized by an array of cytomorphologies, immunologic marker profiles and genetic abnormalities. This heterogeneous entity differs with regard to the cell of origin, site of involvement, symptomatology and response to the treatment (Furie et al., 2003). Evidences suggest that distinctive variants of multicyclic CHOP chemotherapy may perk up the treatment outcome in intermediate grade NHL. The preference of a dose depends on

\footnotetext{
*Corresponding Author:

Dr. Ambili Remesh, Associate Professor

Department of Pharmacology

Dr. Somervell Memorial CSI Medical College

Karakonam, Trivandrum, Kerala, India

E-mail: ambiliremesh@yahoo.com

Contact No.: +91-9497011358
}

the age, performance status, marrow reserve, renal and hepatic functions.

The studies and discovery of new drug entities (Rao et al., 2012; Singh et al., 2012) and novel formulations as well as the adverse reports on conventional medications are compelling the US Food and Drug Administration (US-FDA) and other independent drug safety endeavors to perform their own analyses about potential adverse effects of various drugs through the post-market surveillance efforts. In this line, the French had coined the word 'pharmacovigilance' which means 'study of the undesirable effect of drugs'. The word pharmacovigilance' was derived from the Greek 'pharmakon', a drug or medicine, and from the Latin 'vigilans', watchful or careful. The WHO defined Pharmacovigilance as 'the science and activities relating to the detection, assessment, understanding and prevention of drugrelated adverse effects or any other possible risks (WHO, 2002). Therefore, this study was designed to scrutinize the spectrum of toxicities following six cycles of CHOP chemotherapy regimen in subjects with intermediate grade NHL and to assess the 


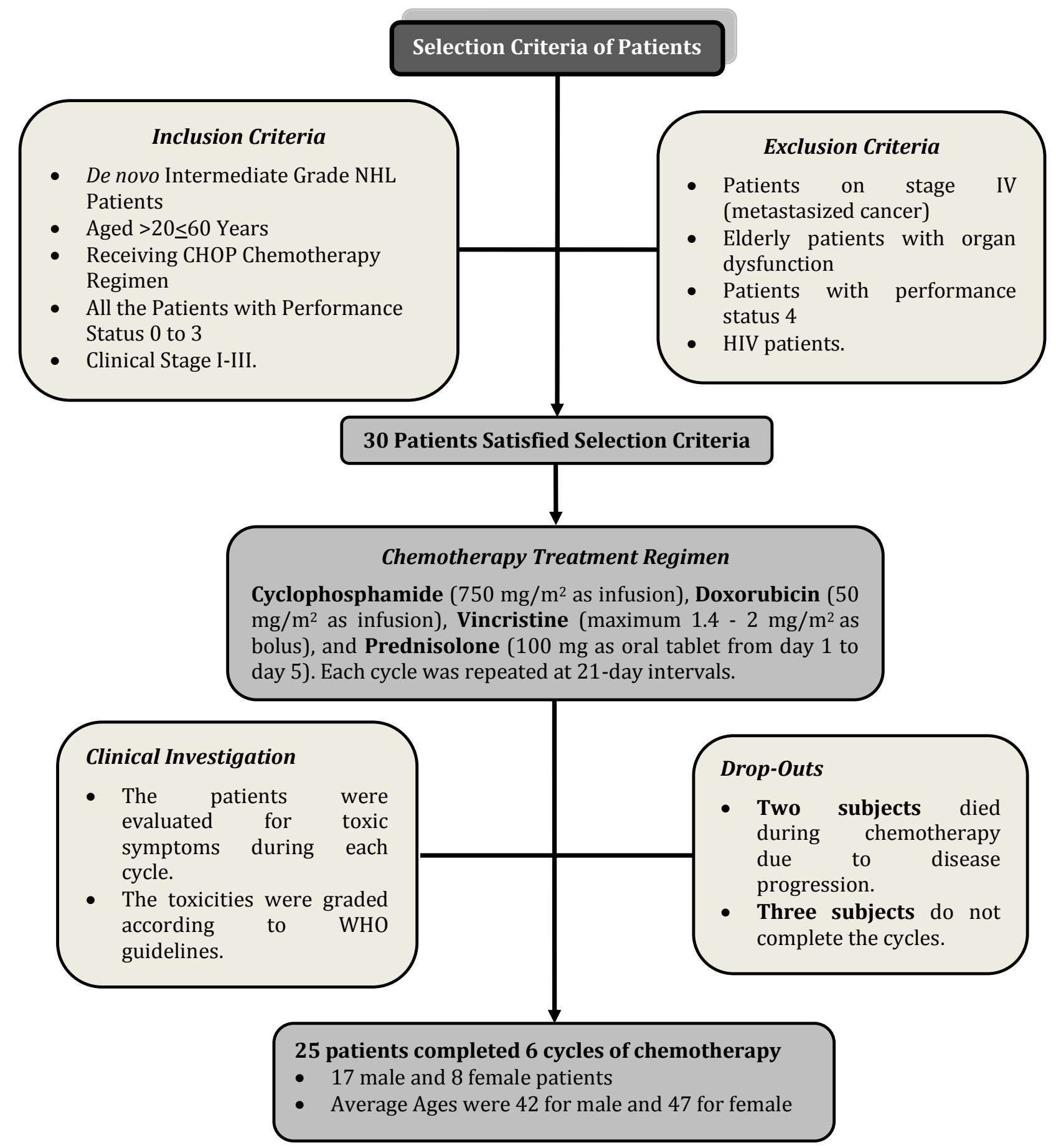

Figure 1: A Schematic Representation of the Clinical Study.

variation of the severity of these toxicities with each cycle of chemotherapy and grade them according to WHO guidelines.

\section{MATERIAL AND METHODS}

This clinical study is a randomized prospective trial conducted on de novo intermediate grade NHL patients, at the Department of Medical Oncology, Regional Cancer Centre (RCC), Trivandrum, India, after obtaining sanction from Institutional Review Board and Scientific Review Committee. All the patients gave their written informed consent prior to entry into the protocol. All the eligible candidates underwent baseline clinical investigations prior to 
chemotherapy. A schematic representation of the clinical study is depicted in Figure 1.

The subjects were free to use $\mathrm{H}_{2}$-receptor antagonists and antacids when needed. All the subjects with chemotherapy underwent clinical investigation at day 10. The patients were examined for toxic symptoms during each cycle and the toxicities were graded according to WHO guidelines (Perry, 1992). In our study, the performance status was graded as per the Eastern Cooperative Oncology Group (ECOG)/ Zubrod's and Karnofsky's scales (Buccheri et al., 1996; Socinski et al., 2003).

\section{RESULTS AND DISCUSSION}

According to the SEER Cancer Statistics Review 1975-2009, the National Cancer Institute, USA estimated that 70,130 men and women (38,160 men and 31,970 women) will be diagnosed with and 18,940 men and women will die of non-Hodgkin lymphoma in 2012 (Howlader et al., 2011). In spite of substantial progress in the understanding of the molecular biology of the NHLs, their precise etiology remains obscure. Moreover, the toxicity profile of CHOP therapy is being evaluated for several decades especially in developing countries like India. Though modern therapeutics options like Rituximab has opened the way for better treatment modalities, the affordability of such therapy in India is still a matter a debate. Hence, a reappraisal of the conventional CHOP therapy is required to ascertain the tolerance, resistance or other pharmacotoxicological outcomes of these drugs in Indian race which in turn throws light on the requirement of any treatment variations with respect to the dose or combination in the chemotherapy regimen.

The CHOP regimen which comprises of cyclophosphamide, doxorubicin, vincristine and predisolone was considered as the gold standard therapy for NHL patients. Cyclophosphamide and doxorubicin are well known to produce hepatic, renal and cardiac toxicities apart from common hematological and bone marrow toxicities. A recent study had shown that R-CHOP therapy causes severe liver damage including the break out of occult hepatitis (Ahmad et al., 2011). In line with that, in our study also the patients developed liver abnormalities manifested by increased serum transaminases, alkaline phosphatase, bilirubin and remarkably hepatomegaly (Figure 2-5). The CHOP therapy though increased the performance status, produced progressive liver damage in the 6 cycles. Also it was observed that the serum levels of transaminases, phosphatase and bilirubin went upto the levels grade $3 \& 4$ toxicities whereas the hepatomegaly did not progress beyond grade 2 in any of the patient till $6^{\text {th }}$ cycle. The increase in liver damage and subsequent release \& increase of serum hepatic biomarkers may be due to the free radical generation by the chempotherapeutics during their metabolism in the liver (Ferreira et al., 2008).

Although, weight loss was found to be increased in few patients (Figure 6), it could be understood that the weight loss did not progress beyond grade 3 or 4 and hence it is due to the disease progression (Friedman et al., 2011) and not due to the toxicity of $\mathrm{CHOP}$ therapy. Alopecia is one of the well established and distressing adverse effects of cancer chemotherapy which occurs due to the misidentification and suppression of rapidly growing hair follicular cell growth by these chemotherapeutics (Kargar et al., 2011). In corroboration with that, in our study also CHOP chemotherapy caused upto grade 2 alopecia in $32 \%$ of the subjects and grade 1 alopecia in $20 \%$ of the patients (Figure 7 ). Hildebrandt et al (2000) reported that CHOP therapy causes dysphagia as observed in our study where the patients reported upto grade 4 dysphagia (Figure 8). In this view, Wang et al., (2000) reported a transient esophageal motor dysfunction with dysphagia in a 62-year-old man receiving vincristine-containing chemotherapy for nonHodgkin's lymphoma. As reported in a recent study by Brunello et al, 2011, a marginal increase in the glycemic levels were observed in the present study (Figure 9). Dysuria was a minor complaint in many and none had dysuria of grade 3 (Figure 10). Alteration in serum creatinine was observed from the first cycle onwards but in none of the subjects, grade 3 or 4 toxicity observed (Figure 11). Though hypotension was not observed as a major toxic manifestation, only one patient showed hypotension which interrupted the chemotherapy (Figure 12). Also, another study had shown that CHOP chemotherapy did not show any persistent low blood pressure (Manolopoulos et al., 2009). 


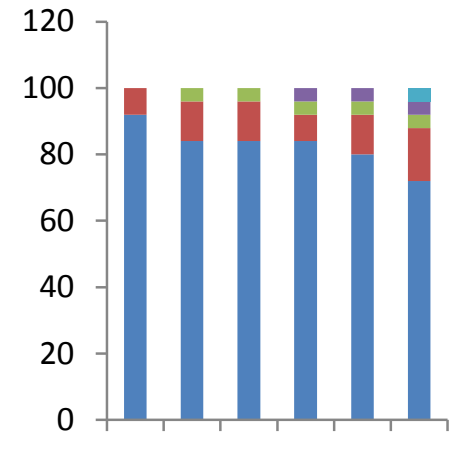

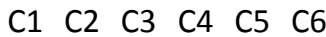

Figure 2: Percentage distribution of Bilirubin and grades in NHL patients.

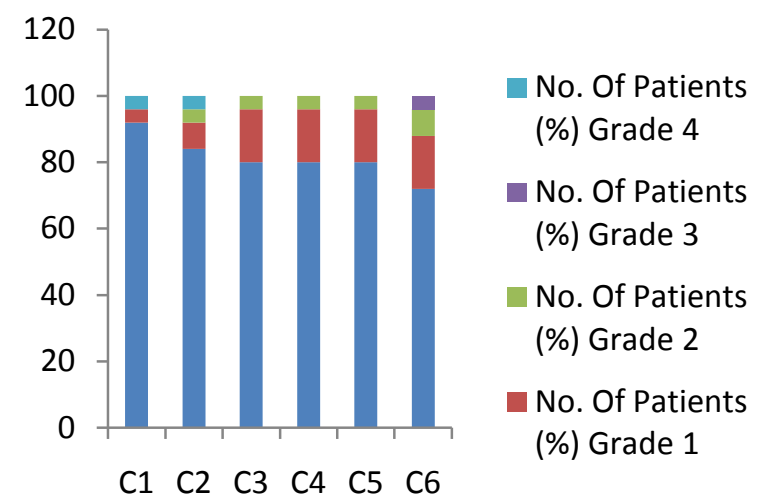

Figure 4: Percentage distribution of serum alkaline phosphatase and grades in NHL patients.

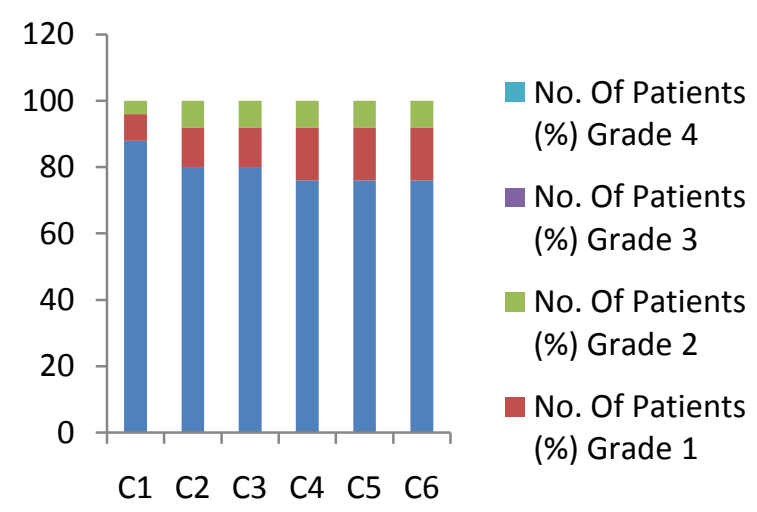

Figure 6: Percentage distribution of weight loss and grades in NHL patients.

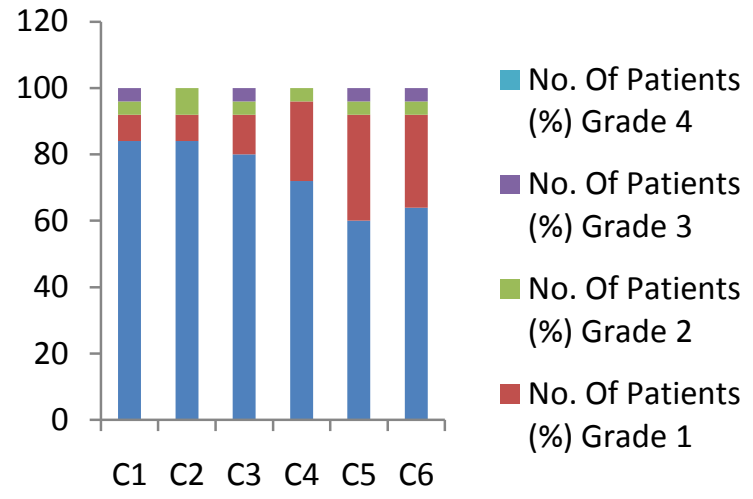

Figure 3: Percentage distribution of serum transaminase and grades in NHL patients.

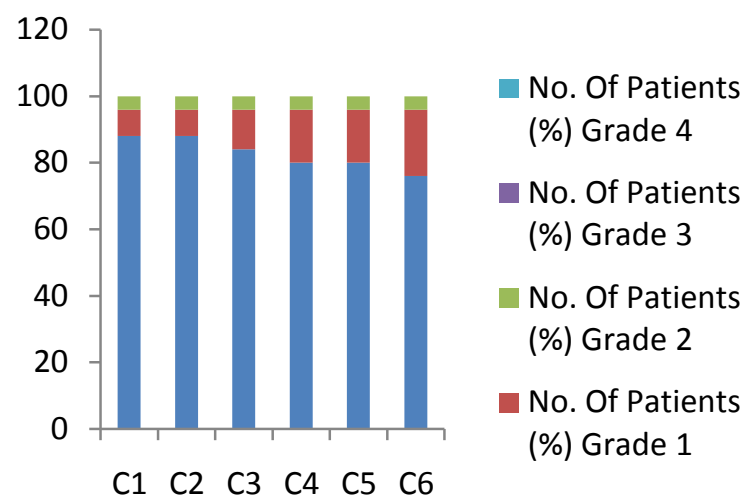

Figure 5: Percentage distribution of hepatomegaly and grades in NHL patients.

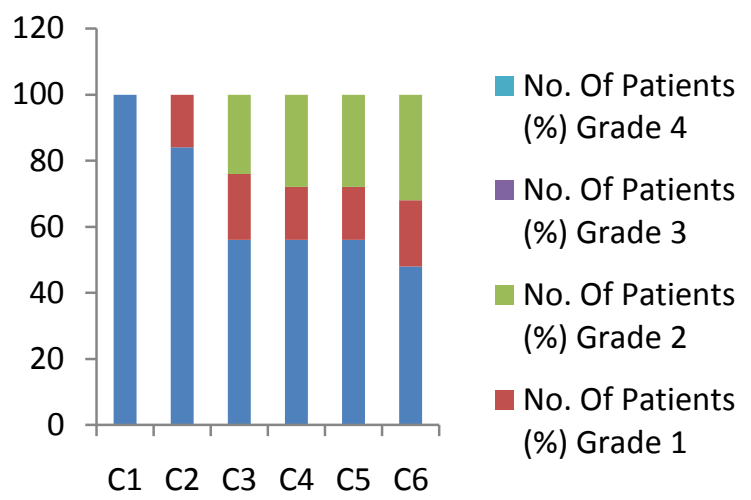

Figure 7: Percentage distribution of alopecia and grades in NHL patients. 


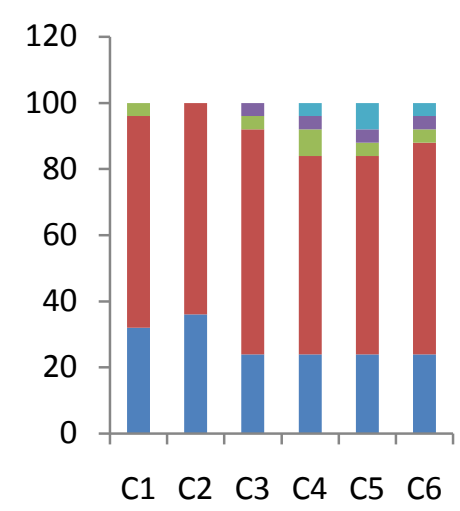

Figure 8: Percentage distribution of dysphagia and grades in NHL patients.

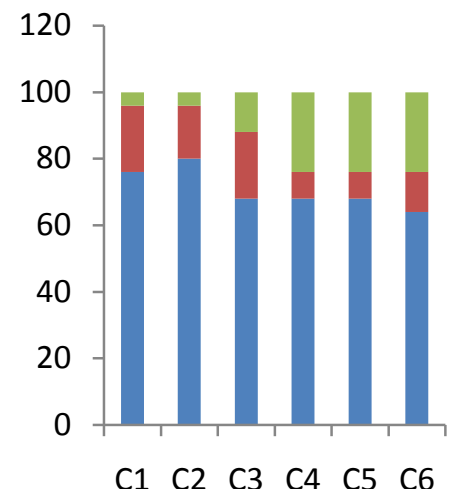

Figure 10: Percentage distribution of dysuria and grades in NHL patients.

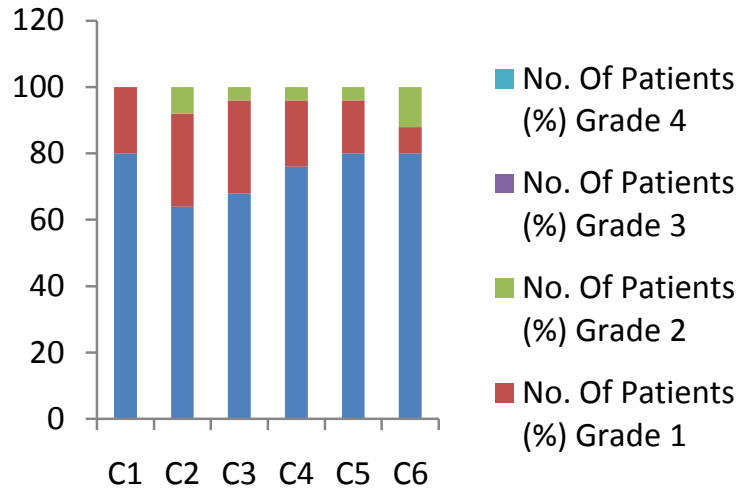

Figure 9: Percentage distribution of hyperglycemia and grades in NHL patients.

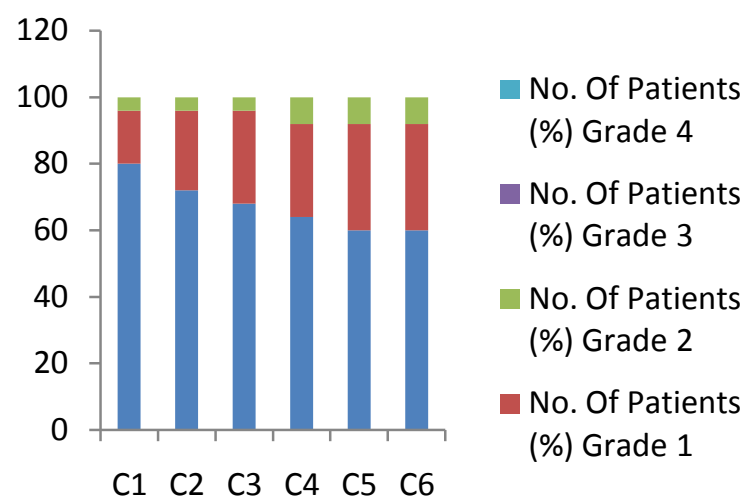

Figure 11: Percentage distribution of creatinine and grades in NHL patients.

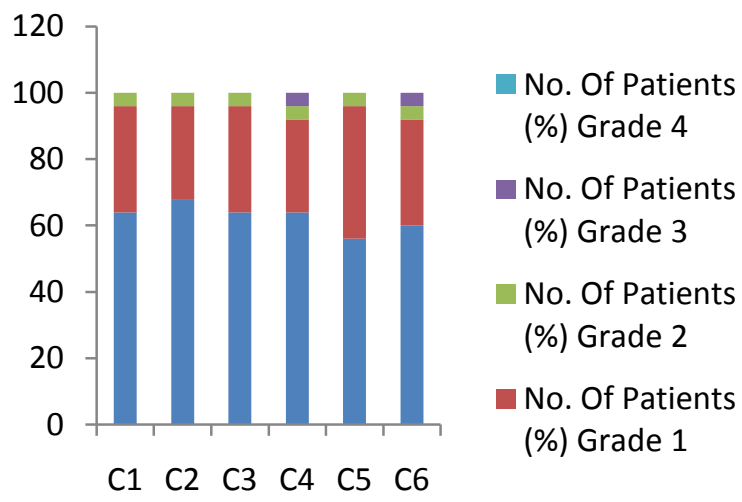

Figure 12: Percentage distribution of hypotension and grades in NHL patients. 


\section{CONCLUSION}

To conclude, it has been observed that in this study, combination chemotherapy with $\mathrm{CHOP}$ protocol was well tolerated by majority of the subjects with a significant control upon the disease progression. However, large pool of subjects must be included in future clinical trials to validate this research outcome.

\section{REFERENCES}

Ahmad, S., Le, V. and Ali, T. (2011). Occult Hepatitis B FlareUp after Chemotherapy Treatment in HBsAg Negative Patient. Int J Clin Med., 2, 621-623. [DOI]

Brunello, A., Kapoor, R. and Extermann, M. (2011). Hyperglycemia during chemotherapy for hematologic and solid tumors is correlated withincreased toxicity. Am J Clin Oncol., 34:292-296. [DOI]

Buccheri, G., Ferrigno, D. and Tamburini, M. (1996) Karnofsky and ECOG performance status scoring in lung cancer: a prospective, longitudinal study of 536 patients from a single institution, Eur. J. Cancer, 32A (7): 1135-1141. [DOI]

Ferreira, A.L.A, Matsubara, L.S. and Matsubara B.B. (2008). Anthracycline-Induced Cardiotoxicity. Cardiovasc Hematol Agents Med Chem, 6: 278-281. [DOI]

Friedman, M.S., Subbiah, I.M., Arora, G., Brodsky, G. and Sood, V. (2011). Diffuse Large B-cell Lymphoma Presenting with Obstructive Jaundice from a Biliary Stricture: A Case Report and Review of Literature. Prac Gastroenterol, 43-48. [Link]

Furie, B., Cassileth, P.A., Atkins, M.B., and Mayer, R.J., (2003). Clinical hematology and oncology: presentation, diagnosis, and treatment: Chapter 66, (First Edition) Churchill Livingstone, Philadelphia, pp: 636-647.

Hildebrandt, G., Holler, E., Woenkhaus, M., Quarch, G., Reichle, A., Schalke, B. and Andreesen R. (2000). Acute deterioration of Charcot-Marie-Tooth disease IA (CMT IA) following $2 \mathrm{mg}$ of vincristine chemotherapy. Ann Oncol. ; 11: 743-747. [DOI]

Howlader, N., Noone, A.M., Krapcho, M., Neyman, N., Aminou, R., Altekruse, S.F., Kosary, C.L., Ruhl, J., Tatalovich, Z., Cho, H., Mariotto, A., Eisner, M.P., Lewis, D.R., Chen, H.S., Feuer, E.J. and Cronin, K.A. (eds). SEER Cancer Statistics Review, 1975-2009 (Vintage 2009

Populations), National Cancer Institute. Bethesda, MD, [Link] , based on November 2011 SEER data submission, posted to the SEER web site, 2012.
Kargar, M., Sarvestani, R.S., Khojasteh, H.N. and Heidari, M.T., (2011). Efficacy of penguin cap as scalp cooling system for prevention of alopecia in patients undergoing chemotherapy. J Adv Nurs, 67: 2473-2477. [DOI]

Manolopoulos, L., Gomatos, I.P., Leandros, E., Alevizos, L., Georgiou, N., Giotakis, J. and Ferekidis, E. (2009). Use of Rituximab in Combination with Conventional Chemotherapy for the Treatment of Non-Hodgkin's Lymphoma of the Head and Neck. In vivo. 23: 475-478.

Perry, M.C. (1992) The Chemotherapy Source Book, Appendix: WHO toxicity guidelines, in Perry MC (ed), Williams \& Wilkins, Baltimore, pp: 1132-1144.

Rao, P.S., Navinchandra, S., Jayaveera, K.N. (2012). An important spice, Pimenta dioica (Linn.) Merill: A Review. International Current Pharmaceutical Journal, 1(8): 221225. [DOI]

Singh N, Pandurangan A, Rana K, Anand P, Ahmad A, Tiwari AK (2012), Benzimidazole: A short review of their antimicrobial activities, International Current Pharmaceutical Journal, 1(5): 119-127. [DOI]

Socinski, M.A., Morris, D.E., Masters G.A. and Lilenbaum R. (2003). American College of Chest Physicians, Chemotherapeutic management of stage IV non-small cell lung cancer, Chest, 123 (1 Suppl): 226S-243S. [DOI]

van de Schans, S.A., Gondos, A., van Spronsen, D.J., Rachtan, J., Holleczek, B., Zanetti, R., Coebergh, J.W., JanssenHeijnen, M.L. and Brenner, H. (2011). Improving relative survival, but large remaining differences in survival for non-Hodgkin's lymphoma across Europe and the United States from 1990 to 2004, J. Clin. Oncol. 29 (2): 192-199. [DOI]

Wang, W.S., Chiou, T.J., Liu, J.H., Fan, F.S., Yen, C.C. and Chen, P.M. (2000) Vincristine-induced dysphagia suggesting esophageal motor dysfunction: a case report. Jpn J Clin Oncol. 30:515-518. [DOI]

WHO (2002). The importance of pharmacovigilance: safety monitoring of medicinal products, Chapter 2, A Short History of Involvement in Drug Safety Monitoring by WHO. The World Health Organization, pp: 5-7. 